\title{
Combination Prevention among youngsters of key populations: a Brazilian experience through peer-to-peer education to promote sexual health
}

Diego Agostinho Calixto, ${ }^{1}$ Carina Bernardes Sousa, ${ }^{1}$ Liliana Pittaluga,${ }^{1}$ Gilvane Casimiro da Silva, ${ }^{1}$ Adele Schwartz Benzaken, ${ }^{1}$ Ministry of Health's Department of Surveillance, Prevention and Control of STIs, HIV/AIDS and Viral Hepatitis, Brasília, Brazil.

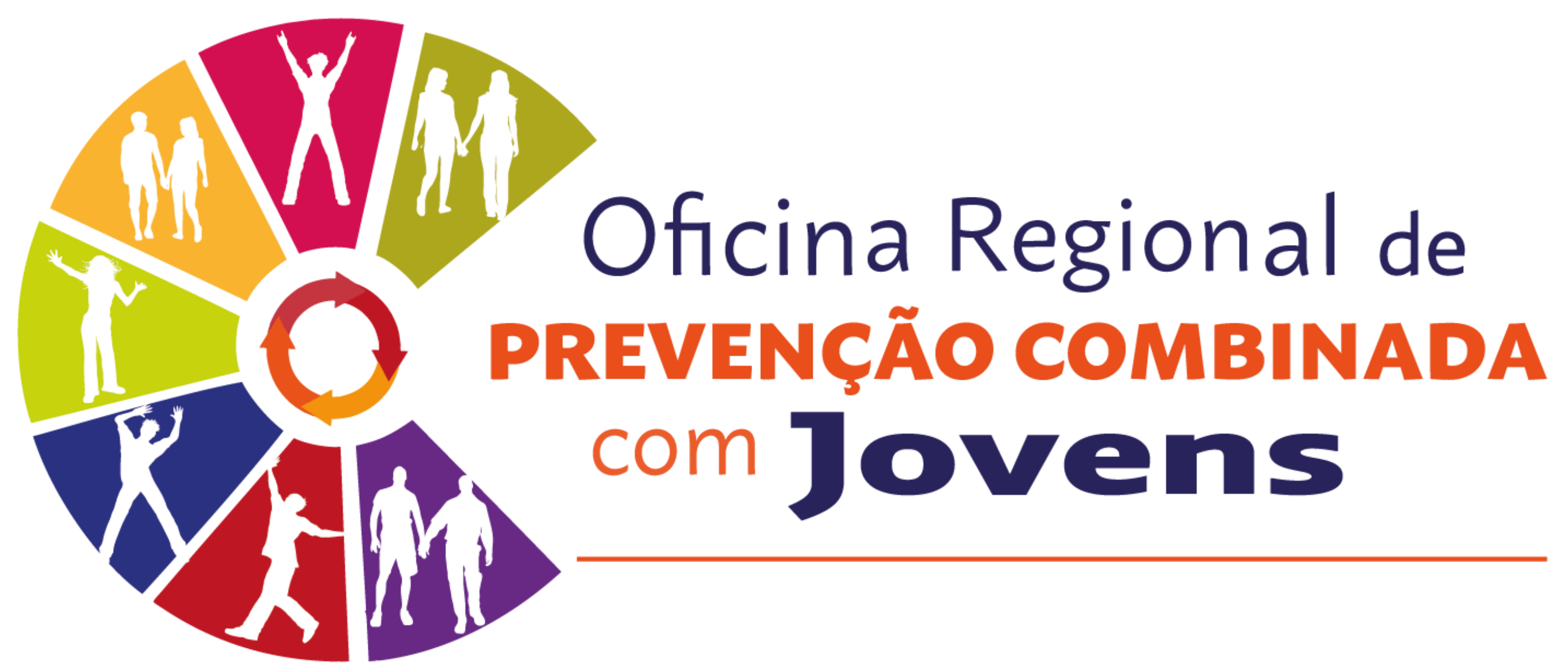

Background: Young people represent $40 \%$ of new HIV infections worldwide. In Brazil, according to the 2017 Epidemiological Report, youngsters aged 15 to 19 accounted for $44.1 \%$ of new HIV cases. In order to understand the specific context of the HIV epidemics in this population, we need a focuses on their specificities and vulnerability contexts. Combination Prevention Workshop with the participation of young key populations (YKP) was marked by an assertive strategy through peer-to-peer education, emphasizing approaches to enhance sexual health through the exchange of experiences. The purpose of this study is to provide a snapshot of Combination Prevention among YKP, based on the information collected through this strategy.

Methods: The Combination Prevention Workshop with YKP is an initiative launched by the Ministry of Health of Brazil in 2017. Six workshops have been conducted, qualifying 380 young people all over the national territory, to act as peer educators for Combination Prevention in their communities.

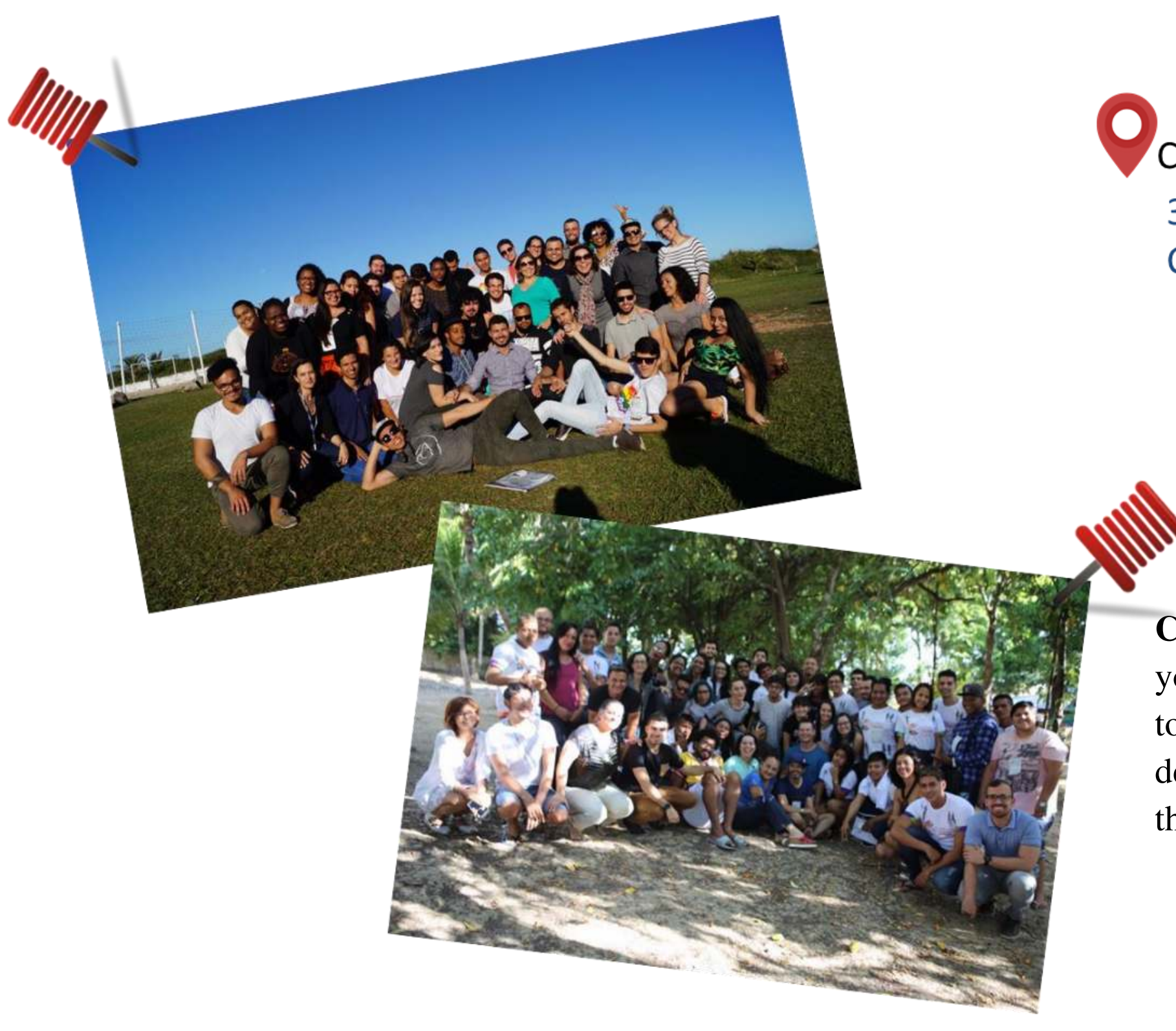

Results: This strategy has helped expanding knowledge and acceptance of Combination Prevention and sexual health by YKP, paving the way for approaches that are coherent with their specificities and vulnerabilities. Besides sharing experiences, this strategy has also helped young people deconstructing biased ideas and discriminatory attitudes associated with sexual orientation, gender identity, use of alcohol and other drugs, sex work, skin color, race or culture, living with HIV, and people with disabilities, among others.

Cities that host the workshops 380 young people trained on Combination Prevention

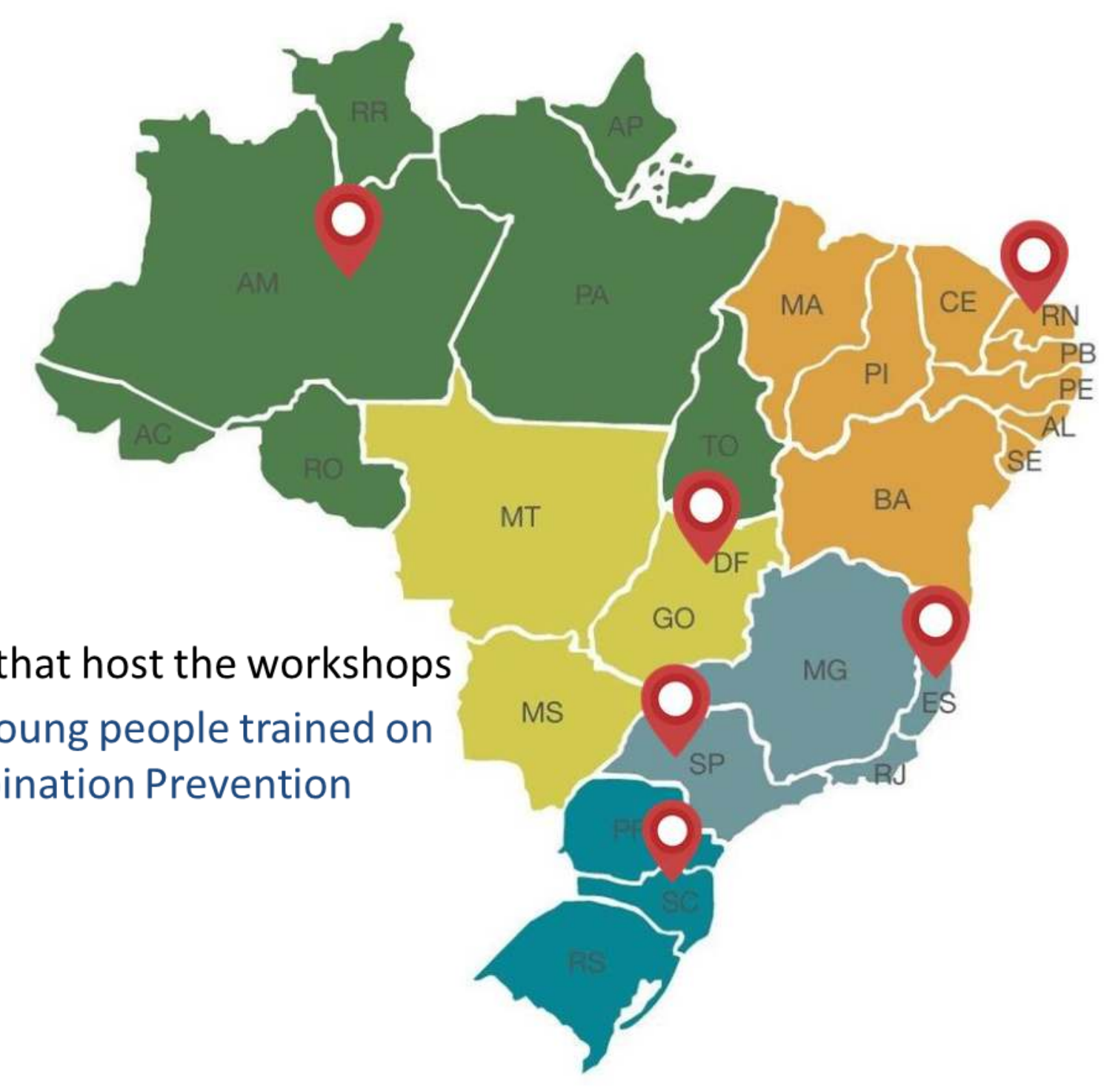

Conclusions: Peer education has shown to improve quality of life of young people. Through this strategy, qualified youngsters have learned to use their knowledge on Combination Prevention and sexual health to develop in their communities, take initiatives and, above all, commit themselves to their own health and to their local 